\title{
Does probiotics reduce crying time in infantile colic in exclusively breastfed babies? A comprehensive meta-analysis
}

\author{
K H Shabuj ' , C K Shaha ${ }^{2}$, J Hossain ${ }^{3}$
}

\begin{abstract}
Background : Infantile colic is one of the common pediatric problems that Pediatricians encounter in practice. This condition is associated with excessive crying in healthy thriving infants over a regular period during the day and is sustained for the first few months of life. It has been historically described as irritable or compulsive crying or paroxysmal fussing with multifactorial etiology. Although it affects $5-19 \%$ of young infants, it remains a frustrating problem for parents and caregivers, because it is difficult to treat and may result in significant psychosocial consequences. Different treatment options are available with no definitive and conclusive benefit. In this metaanalysis, we pooled relevant data regarding the use of probiotics in infantile colic and tried to find out any beneficial effect thereof.
\end{abstract}

Objective : To evaluate the efficacy of probiotics supplementation in the reduction of the duration of crying time in infantile colic.

Method : It is a comprehensive meta-analytic study where literature searches were conducted in the Pubmed using end note package and Google scholar. Randomized controlled trials those enrolled healthy, term breastfed infants with infantile colic were included for analysis. After meticulous scrutiny for methods and study population, five studies with a total of 384 breastfed infants were selected for final analysis. All these studies used Lactobacillus reuteri DSM 17938 as probiotics in the therapeutic intervention and supplementation of this was compared to placebo.

Result : Analysis of pooled data showed that at Initiation of therapy mean difference of crying time 0.29 minutes; $95 \% \mathrm{Cl}-6.61,7.20, \mathrm{p}=0.89$, after 3 weeks of probiotics therapy mean difference of crying time $-30.64,95 \% \mathrm{Cl}$ $34.48,-26.81, p=0.0001$.

${ }^{1}$ Dr. Kamrul Hassan Shabuj Assistant Professor Neonatology, Bangabandhu Sheikh Mujib Medical University (BSMMU), Dhaka

2

Dr. Chandan Kumar Shaha Assistant Professor Dept. of Pediatrics Sir Salimullah Medical College Dhaka

3 Dr. Jesmin Hossain Assistant Professor Pediatric Cardiology National Heart Foundation Hospital, Mirpur, Dhaka

\section{Correspondence}

Dr. Kamrul Hassan Shabuj Assistant Professor

Neonatology, Bangabandhu

Sheikh Mujib Medical University (BSMMU), Dhaka

e-mail: shabuj619@yahoo.com

Conclusions : Probiotics use for 3 weeks significantly reduced the duration of crying time in exclusively breastfed infants suffering from infantile colic.

Keywords : Infantile colic, Probiotics, Meta-analysis, Lactobacillus reuteri

DOI: http://dx.doi.org/10.3329/nimcj.v9i1.35919

Northern International Medical College Journal Vol. 9 No. 1 July 2017, Page 245-247

\section{Introduction}

Infantile colic is a common pediatric condition, which causes significant parental distress. ${ }^{1}$ It is defined as paroxysmal, excessive, and inconsolable crying without an identifiable cause in an otherwise healthy newborn infant and is common in the first 3 months of life. ${ }^{2,3}$ The classic definition of infantile colic is based on the rule of 3's:

- fussy crying that lasts for 3 hours per day

- for 3 days per week and

- for a minimum of 3 weeks. ${ }^{4}$
Colic affects $3-28 \%$ of infants, causing considerable stress and concern for parents. ${ }^{5}$ The pathogenesis of the condition remains elusive, although evidence suggests multiple independent causes. ${ }^{3}$ Despite forty years of research, the etiology of infantile colic remains non-conclusive. The current literatures suggest several causative mechanisms such as behavioral, food allergy and hypersensitivity, immaturity of gut function and dysmotility as the probable etiologies. $^{6-8}$ The role of an aberrant intestinal microflora has recently been reproposed to affect gut motor function and gas production that lead to colicky behavior. ${ }^{9}$ 
We performed an extensive literature search on published relevant articles to find out the effectiveness of probiotics in the reduction of the duration of crying time in healthy, term, exclusively breastfed infant with infantile colic.

\section{Methods}

In this meta-analysis we aimed to identify whether the probiotic Lactobacillus reuteri DSM 17938 was effective in the management of infant colic. In this study, we analyzed the reduction of duration of infant crying time at 21 days postintervention.But we did not analyze the treatment success (at least $50 \%$ reduction in crying time from baseline) at 21 days post intervention.

We specifically included all those studies which were doubleblinded RCT and were done on exclusive breastfed infant using probiotics Lactobacillus reuteri DSM 17938 and placebo consisting of same taste and color but not containing Lactobacillus reuteri DSM 17938.

We searched randomized controlled trials by identification of published papers through the Pubmed online databases and Google scholar. We found no research work from Bangladesh which evaluated effectiveness of probiotics in the management of infantile colic.

All analysis in this meta-analysis were based on previous published studies; thus no ethical approval or patient consent was required. Literature search and selected articles shown in the following flow chart (Figure-1).

\section{Figure 1: Study Flow Chart}

Pub med and Google scholar $\leftarrow(\mathrm{N}=69)$ Probiotics and Infantile colic
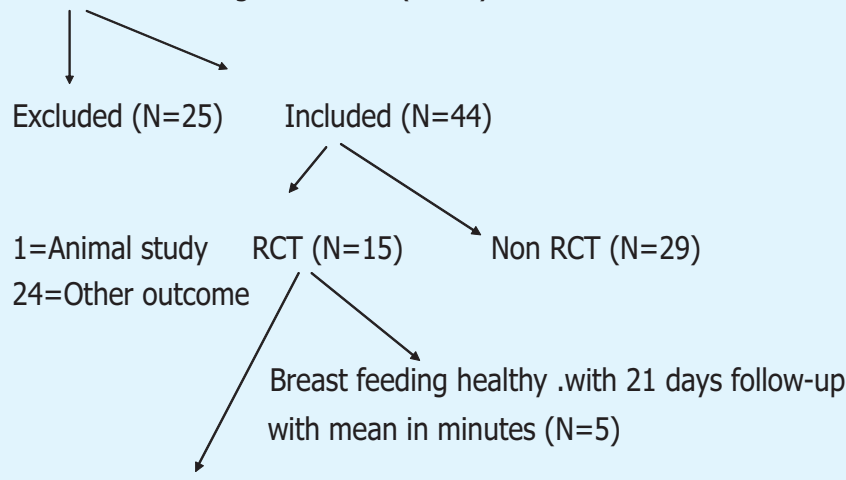

Formula + Simithecone not followed 21 days,

no mean, interquartile range $(\mathrm{N}=10)$

\section{Data analysis}

Abstracting data from published randomized trials, estimates of the SDs in crying time (min/day) at baseline and day 21 were collected and pooled and data were analyzed by using Rev Man and comprehensive meta-analysis package 7.1 (30 days free trial). We presented the data using Forest plot.

\section{Main Results}

We identified five studies for inclusion in our meta-analysis, representing a total of 384 infants. There were three further studies that we could not find information about and we identified three other ongoing studies. [of the five included studies, four were suggestive of a beneficial effect and one found no evidence of reduction of duration of crying time at 3 weeks of therapy]. Tests for heterogeneity imply that there may be some underlying difference between this study and the other four studies (Table I).

Five studies measured daily hours of crying and these data were combined, suggesting that manipulative therapies had a significant effect on infant colic-reducing average crying time by 3 weeks of therapy with probiotics in unit minute/day (mean difference (MD) -30.64; 95\% confidence interval (CI) -34.48 to 26.83. (Table-II).Test for overall effect size is significant $(\mathrm{z}=15.66 p<0.00001)$.

\section{Discussion}

In this meta-analytic study we pooled data of randomized trials addressing the efficacy of probiotics in infantile colic after intervention for a period of three weeks. A significant effect of L.reuteri supplementation strain DSM 17938 in shortening crying times. However, the effect in the probiotics group was more pronounced.

Our meta-analysis included currently available, high quality studies which were published in world renowned journals. However; our overall conclusion is compromised by the small number of enrolled infants despite the common prevalence of infantile colic, and the heterogeneity of included studies that assessed crying time. This heterogeneity can be explained by the clear imbalance of both groups at baseline, in the study reported by Savino et al. ${ }^{13}$ The probiotic group started with significantly longer crying times that quickly moved to a profoundly positive benefit after 21 days of treatment. Most of the included subjects were exclusively breast fed which limits the generalization of the findings to formula fed infants.

The earlier trial by Savino et al. ${ }^{14}$ involved the use of simethicone in the placebo arm. This may have decreased the magnitude of the effect of probiotics unless the effect of simethicone was not indifferent to placebo. One well recognized limitation of all studies on infant colic is the need for a more objective way of measuring duration of crying rather than relying on the parents' compliance to establish this outcome.

Computer recordings of crying episodes comprise a more concrete form of assessment and should be considered for future studies. Two of the included trials were supported by the manufacturer of the probiotic strain under study, which raises the possibility of bias. However, the likelihood is small since the 
Table I: Baseline crying time

\begin{tabular}{|c|c|c|c|c|c|c|c|c|c|}
\hline \multirow[b]{2}{*}{ Study or Subgroup } & \multicolumn{3}{|c|}{ probiotics } & \multicolumn{2}{|c|}{ Placebo } & \multirow{2}{*}{ Total } & \multirow[b]{2}{*}{ Weight } & \multirow{2}{*}{$\begin{array}{l}\text { Mean Difference } \\
\text { IV, Fixed, } 95 \% \mathrm{Cl}\end{array}$} & \multirow{2}{*}{$\begin{array}{l}\text { Mean Difference } \\
\text { IV, Fixed, } 95 \% \mathrm{Cl}\end{array}$} \\
\hline & Mean & SD & Tota & Mean & SD & & & & \\
\hline chau K 2015 & 125.5 & 88.8 & 24 & 116.5 & 83.81 & 24 & $2.0 \%$ & $9.00[-39.85,57.85]$ & \\
\hline Savino 2007 & 212.5 & 29.1 & 41 & 213 & 29.8 & 42 & $29.7 \%$ & $-0.50[-13.17,12.17]$ & \\
\hline Savino 2010 & 370 & 30 & 25 & 300 & 375 & 21 & $0.2 \%$ & $70.00[-90.82,230.82]$ & \\
\hline Sung 2014 & 175 & 115 & 67 & 166 & 93 & 60 & $3.6 \%$ & $9.00[-27.22,45.22]$ & \\
\hline Szajewska 2012 & 240 & 17.3 & 40 & 240.3 & 21.7 & 40 & $64.5 \%$ & $-0.30[-8.90,8.30]$ & \\
\hline Total $(95 \%$ Cl) & & & 197 & & & 187 & $100.0 \%$ & $0.29[-6.61,7.20]$ & \\
\hline \multicolumn{9}{|c|}{$\begin{array}{l}\text { Heterogeneity: } \mathrm{Chi}^{2}=1.10, \mathrm{df}=4(P=0.89) ;\left.\right|^{2}=0 \% \\
\text { Test for owerall effect: } Z=0.08(P=0.93)\end{array}$} & $\begin{array}{ccccc}-50 & -25 & 0 & 25 & 50 \\
\text { Favours [experimental]] } & \text { Favours [contro] }\end{array}$ \\
\hline
\end{tabular}

(Studies included: Chau $\mathrm{K}^{10}$, Savino ${ }^{14}$, Savino $0^{13}$, Sung ${ }^{12}$, Szajewska ${ }^{17}$ )

Table II : Crying time after 3 weeks use of probiotics

\begin{tabular}{|c|c|c|c|c|c|c|c|c|c|}
\hline \multirow[b]{2}{*}{ Study or Subgroup } & \multicolumn{2}{|c|}{ probiotic } & \multicolumn{4}{|c|}{ placebo } & \multicolumn{2}{|r|}{ Mean Difference } & \multirow{2}{*}{$\begin{array}{l}\text { Mean Difference } \\
\mathrm{IV} \text {, Fixed, } 95 \% \mathrm{Cl} \\
\end{array}$} \\
\hline & Mean & SD & Total & Mean & SD & Total & Weight & IV, Fixed, $95 \% \mathrm{Cl}$ & \\
\hline Chau k 2015 & 104 & 103.01 & 24 & 67 & 82.52 & 28 & $0.6 \%$ & $37.00[-14.31,88.31]$ & \\
\hline savino 2007 & 80.5 & 30.3 & 41 & 147.8 & 50.6 & 42 & $4.6 \%$ & $-67.30[-85.19,-49.41]$ & 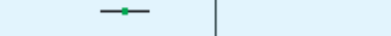 \\
\hline Savino 2010 & 35 & 21.3 & 25 & 90.37 & 37 & 21 & $4.6 \%$ & $-55.37[-73.26,-37.48]$ & \\
\hline Sung 2014 & 76 & 82 & 67 & 79 & 77 & 60 & $1.9 \%$ & $-3.00[-30.66,24.66]$ & - \\
\hline Szajewska 2012 & 75 & 8.7 & 40 & 103.5 & 9.9 & 40 & $88.3 \%$ & $-28.50[-32.58,-24.42]$ & \\
\hline \multirow{2}{*}{\multicolumn{6}{|c|}{$\begin{array}{l}\text { Heterogeneity: Chi }{ }^{2}=35.03, \mathrm{df}=4(\mathrm{P}<0.00001) ; \mathrm{I}^{2}=89 \% \\
\text { Test for overall effect: } Z=15.66(P<0.00001)\end{array}$}} & 191 & $100.0 \%$ & $-30.66[-34.50,-26.83]$ & 1 \\
\hline & & & & & & & & & $\begin{array}{ccccc}-100 & -50 & 0 & 50 & 100 \\
\text { Favors probiotics } & \text { Favors placebo }\end{array}$ \\
\hline
\end{tabular}

trials were fully investigator-initiated and data controlled with transparent disclosure of potential conflicts of interest by the respective authors. ${ }^{15,16}$ Recently few studies have addressed the role of changing intestinal microbiota in the pathogenesis of colic. Colicky infants were found to have increased colonization by coliforms especially E.coli and decreased and altered colonization. ${ }^{17}$

\section{Limitations of the study}

With only a sample of 384 infants, we have too few data to reach any definitive conclusions about safest use of probiotics. During inclusion of studies we have substantial evidence of heterogeneity $\left(\mathrm{I}^{2}=89 \%\right)$ and to avoid heterogeneity we do not do any sub group analysis.

\section{References}

1. Illingworth RS. Infantile colic revisited. Arch Dis Child 1985;60:981-5.

2. Roberts DM, Ostapchuk M, O'Brien JG. Infantile colic. Am Fam Physician 2004;70(4): 735-740

3. Savino F. Focus on infantile colic. Acta Paediatr 2007; 96(9):1259-1264

4. Wessel MA, Cobb JC, Jackson EB, Harris GS Jr, Detwiler AC. Paroxismal fussing in infancy,sometimes called "colic."Pediatrics 1954;14(5):421- 435.

5. Keefe MR, Kajrlsen KA, Lobo ML, Kotzer AM, Dudley WN. Reducing parenting stress in families with irritable infants. Nurs Res 2006;55(3):198 -205

6. Miller AR, Barr RG: Infantile colic. Is it a gut issue? Pediatr Clin North Am 1991, 38:1407-1423.
7. Treem WR: Infant colic. A pediatric gastroenterologist's perspective. Pediatr Clin North Am 1994, 41:1121-1138.

8. Lucassen PL, Assendelft WJ, Gubbels JW. Effectiveness of treatments for infantile colic: systematic review. BMJ 1998, 316:1563-1569.

9. Gupta SK. Is colic a gastrointestinal disorder? Curr Opin Pediatr. 2002;14(5): 588-592. 10. Chau K, Lau E, GreenbergS,Jacobson S, Yazdani Brojeni P, Verma $\mathrm{N}$, et al. Probiotics for infantile colic: a randomized, double-blind, placebo-controlled trial investigating Lactobacillus reuteri DSM 17938. J Pediatr 2015;166(1):74-8.

11. Savino F, Ceratto S, De Marco A, Cordero di Montezemolo L. Looking for new treatments of Infantile Colic. Ital J Pediatr 2014;40:53.

12. Sung V, Cabana MD, D'Amico F, Deshpande G, Dupont C, Indrio F, et al. Lactobacillus reuteri DSM 17938 for managing infant colic: protocol for an individual participant data meta-analysis. BMJ Open 2014;4(12):e006475.

13. Savino F, Cordisco L, Tarasco V. Lactobacillus reuteri DSM 17938 in infantile colic: a randomized, double-blind, placebo-controlled trial. Pediatrics 2010, 126:e526-e533

14. Savino F, Pelle E, Palumeri E. Lactobacillus reuteri (American Type Culture Collection Strain 55730) versus simethicone in the treatment of infantile colic: a prospective randomized study. Pediatrics 2007, 119:e124-e130.

15. Ross JS, Gross CP, Krumholz HM. Promoting transparency in pharmaceutical industry-sponsored research. Am J Public Health 2012,102:72-80.

16. Dunn AG, Gallego B, Coiera E. Industry influenced evidence production in collaborative research communities: a network analysis. J Clin Epidemiol 2012, 65:535-543.

17. Szajewska H, Gyrczuk E, Horvath A: Lactobacillus reuteri DSM 17938 for the management of infantile colic in breastfed infants: a randomized, doubleblind, placebo-controlled trial. J Pediatr 2013, 162:257-62. 